\title{
Graph operations on Cayley graphs of semigroups
}

\author{
Narges Hosseinzadeh ${ }^{1 *}$, Amir Assari ${ }^{2}$ \\ ${ }^{1}$ Islamic Azad University,Dezful branch, Iran \\ 2 Jundi-Shapur University of Technology, Dezful, Iran \\ ${ }^{*}$ Corresponding author E-mail: narges.hosseinzadeh@gmail.com
}

Copyright (C2014 Narges Hosseinzadeh, Amir Assari. This is an open access article distributed under the Creative Commons Attribution License, which permits unrestricted use, distribution, and reproduction in any medium, provided the original work is properly cited.

\begin{abstract}
Lots of large graphs can be constructed from existing smaller graphs by using graph operations, such as the graph products. Many properties of such large graphs are closely related to those of the corresponding smaller ones.In this paper we consider some operations of Cayley graphs on semigroups.
\end{abstract}

Keywords: graph operation, Cyaley graph, Col-Aut-vertex transitive

\section{Introduction}

Symmetric properties of graphs have been considered by lots of authors in the literature as well as properties of Cayley graphs, see for example [2] and [3]. Cayley graphs of semigroups are also mentioned in some papers such as [5]. Here we discuss about the Cayley graphs of semigroups and talk about some operations such as product of them or complement of it, and finally verify whether the product of Cayley graphs with some special property has the property as well.

Let $\Gamma=(V, E)$ be a simple graph, where $V$ is the set of vertices and $E$ is the set of edges of $\Gamma$. An edge joins the vertex $u$ to the vertex $v$ is denoted by $(u, v)$. The group of automorphisms of $\Gamma$ is denoted by $\operatorname{Aut}(\Gamma)$, which acts on vertices of $\Gamma$. $\Gamma$ is called $X$-vertex transitive if $X \leqslant A u t(\Gamma)$ acts transitively on the set of vertices of $\Gamma$.

Let $G$ be a semigroup and $S$ ba a nonempty subset of $G$. The Cayley graph of $G$ relative to $S$ is denoted by $\Gamma=\operatorname{Cay}(G, S)$ is a graph with vertex set $G$ and $(x, y)$ is an edge of $\Gamma$ if and only if for some $s \in S$ we have $y=s x$. An automorphism $\phi$ of the Cayley graph $\Gamma$ is called colour preserving if $s x=y$ implies $s\left(x^{\phi}\right)=y^{\phi}$. Denote by $\mathrm{ColAut}_{S}(G)$ the set of all colour preserving automorphism of $\Gamma$ and $\Gamma$ is said to be Col-Aut-vertex transitive Cayley graph if it is $\operatorname{ColAut}_{S}(G)$-vertex transitive [5].

Every semigroup $S$ can be converted to a monoid $S^{1}$ which is $S$ if $S$ is a monoid and otherwise is $S \cup\{1\}$ where 1 is an element which is not contained in $S$ with the the extention of definition of multiply by $s .1=1 . s=s \forall s \in S$ and $1.1=1 .[4]$

Many large graphs can be constructed by expanding of small graphs, thus it is important to know which properties of small graphs can be transfered to the expanded one, for example Wang in [7] proved that the lexicographic of vertex transitive graphs is also vertex transitive as well as the lexicographic product of edge transitive graphs. Specapan in [6] Found the fewest number of vertices fo Cartesian product of two graphs whose removal from the 
graph results in a disconnected or trivial graph.Motivated by these we consider five kinds of graph products as the expander graphs which is described below and verify if we can regard the product of them as a Cayley graph of the semigroup which is made by their product underlying semigroup and if the answer is positive does it inherit Col-Aut-vertex property of them.

Definition 1.1. Let $\Gamma_{1}=\left(V_{1}, E_{1}\right)$ and $\Gamma_{2}=\left(V_{2}, E_{2}\right)$ be two graphs. $\Gamma=(V, E)$, the product of them is a graph with vertex set $V=V_{1} \times V_{2}$, and two vertices $\left(u_{1}, u_{2}\right)$ is adjacent to $\left(v_{1}, v_{2}\right)$ in $\Gamma$ if one of the relevant conditions happen depending on the product.

1. Cartesian product. $u_{1}$ is adjacent to $v_{1}$ in $\Gamma_{1}$ and $u_{2}=v_{2}$ or $u_{1}=v_{1}$ and $u_{2}$ is adjacent to $v_{2}$ in $\Gamma_{2}$.

2. Tensor product. $u_{1}$ is adjacent to $v_{1}$ in $\Gamma_{1}$ and $u_{2}$ is adjacent to $v_{2}$ in $\Gamma_{2}$.

3. Strong product. $u_{1}$ is adjacent to $v_{1}$ in $\Gamma_{1}$ and $u_{2}=v_{2}$ or $u_{1}=v_{1}$ and $u_{2}$ is adjacent to $v_{2}$ in $\Gamma_{2}$ or $u_{1}$ is adjacent to $v_{1}$ in $\Gamma_{1}$ and $u_{2}$ is adjacent to $v_{2}$ in $\Gamma_{2}$.

4. Lexicographic. $u_{1}$ is adjacent to $v_{1}$ in $\Gamma_{1}$ or $u_{1}=v_{1}$ and $u_{2}$ is adjacent to $v_{2}$ in $\Gamma_{2}$.

5. Co-normal product. $u_{1}$ is adjacent to $v_{1}$ in $\Gamma_{1}$ or $u_{2}$ is adjacent to $v_{2}$ in $\Gamma_{2}$.

and the complement of a graph $\Gamma=(V, E)$ is denoted by $\Gamma^{C}$ is a graph with the same vertex set and two vertices are adjacent iff they are not adjacent in the original graph $\Gamma$.

\section{Cartesian product}

Through this section we assume $H$ and $K$ are two semigroups, $S$ and $T$ be the subsets of $H$ and $K$ respectively which does not contain the identity and idempotent, if there exisit any. $\Gamma_{1}=\operatorname{Cay}(H, S)$ and $\Gamma_{2}=C a y(K, T)$ and $\Gamma=(V, E)$ be the Cartsian product of them and we denote it by $\Gamma=\Gamma_{1} \square \Gamma_{2}$.

Theorem 2.1. $\Gamma$ the Cartesian product of two Cayley graph of semigroups is also a Cayley graph of the monoid $H^{1} \times K^{1}$ with respect to the subset $\left(\left\{1_{H^{1}}\right\} \times T\right) \cup\left(S \times\left\{1_{K^{1}}\right\}\right)$ of $H^{1} \times K^{1}$.

Proof. $\left(\left(h_{1}, k_{1}\right),\left(h_{2}, k_{2}\right)\right)$ is an edge of $g$ if and only if $h_{1}=h_{2}$ and $k_{2}=t k_{1}$ for some $t \in T$ or for some $s \in S$ we have $h_{2}=s h_{1}$ and $k_{1}=k_{2}$, i.e. for some $\left(1_{H^{1}}, t\right) \in\left\{1_{H^{1}}\right\} \times T$ we have $\left(h_{2}, k_{2}\right)=\left(1_{H^{1}}, t\right)\left(h_{1}, k_{1}\right)$ or for some $\left(s, 1_{K^{1}}\right) \in S \times\left\{1_{K^{1}}\right\}$ we have $\left(h_{2}, k_{2}\right)=\left(s, 1_{K^{1}}\right)\left(h_{1}, k_{1}\right)$, and equivalantly for some $\alpha \in\left(\left\{1_{H^{1}}\right\} \times T\right) \cup\left(S \times\left\{1_{K^{1}}\right\}\right)$, the equiation $\left(h_{2}, k_{2}\right)=\alpha\left(h_{1}, k_{1}\right)$ holds.

Theorem 2.2. Let $\Gamma_{1}$ and $\Gamma_{2}$ be two Col-Aut-vertex transitive Cayley graphs of finite semigroups. Then $\Gamma=(V, E)$ the cartesian product of them is also Col-Aut-vertex transitive Cayley graph.

Proof. Let $\left(h_{1}, k_{1}\right)$ and $\left(h_{2}, k_{2}\right)$ be two arbitrary vertices of the graph $\Gamma$. Since $\Gamma_{1}$ and $\Gamma_{2}$ are Col-Aut-vertex transitive, there exists $\alpha \in \operatorname{ColAut}_{S}(H)$ and $\beta \in \operatorname{ColAut}_{T}(K)$ which sends $h_{1}$ to $h_{2}$ and $k 1$ to $k_{2}$ respectively, implying $(\alpha, \beta)$ sends $\left(h_{1}, k_{1}\right)$ to $\left(h_{2}, k_{2}\right)$. Now we have to show that $(\alpha, \beta)$ is a colour preserving automorphism of the graph $\Gamma$.

If $u_{1}=v_{1}$ and $u_{2}$ and $v_{2}$ are adjacent in $\Gamma_{2}$, since $\beta$ is an automorphism of $\Gamma_{2}$, therefore $(\alpha, \beta)\left(u_{1}, v_{1}\right)$ is also adjacent to $(\alpha, \beta)\left(u_{2}, v_{2}\right)$ in $\Gamma$. Similar argument can be done if $u_{1}$ is adjacent to $v_{1}$ in $\Gamma_{1}$ and $u_{2}=v_{2}$ as well, yields that $(\alpha, \beta)$ is an automorphism of the graph $\Gamma$.

Now suppose for some $(s, t) \in\left(\left\{1_{H}\right\} \times T\right) \cup\left(S \times\left\{1_{K}\right\}\right)$ and $(h, k),\left(h^{\prime}, k^{\prime}\right) \in H \times K$, we have $(s, t)(h, k)=\left(h^{\prime}, k^{\prime}\right)$, then either $s=1_{H}, t \in T$ or $s \in S, t=1_{K}$. Without loss of generality we assume the first case happens and hence $h=h^{\prime}$. Since $\beta \in \operatorname{ColAut}_{T}(K)$ and $t k=k^{\prime}$, we have $t k^{\beta}=k^{\prime \beta}$, implying $(s, t)(h, k)^{(\alpha, \beta)}=\left(h^{\alpha}, k^{\prime \beta}\right)=\left(h, k^{\prime}\right)^{(\alpha, \beta)}=$ $\left(h^{\prime}, k^{\prime}\right)^{(\alpha, \beta)}$, i.e. $(\alpha, \beta)$ is colour preserving.

\section{Tensor product}

Through this section we assume $H$ and $K$ are two semigroups, $S$ and $T$ be the subsets of $H$ and $K$ respectively which does not contain the identity and idempotent, if there exisit any. $\Gamma_{1}=\operatorname{Cay}(H, S)$ and $\Gamma_{2}=C a y(K, T)$ and $\Gamma=(V, E)$ be the tensor product of them and we denote it by $\Gamma=\Gamma_{1} \times \Gamma_{2}$. In the [1], authors proved the following theorems. 
Theorem 3.1. $\Gamma$, tensor product of the graphs $\Gamma_{1}$ and $\Gamma_{2}$ is also a Cayley graph of the semigroup $H \times K$ with respect to the subset $S \times T$ of $H \times K$.

Theorem 3.2. Let $\Gamma_{1}$ and $\Gamma_{2}$ be two Col-Aut-vertex transitive Cayley graphs of finite semigroups. Then $\Gamma=(V, E)$ the tensor product of them is also Col-Aut-vertex transitive Cayley graph.

\section{Strong product}

Through this section we assume $H$ and $K$ are two semigroups, $S$ and $T$ be the subsets of $H$ and $K$ respectively which does not contain the identity and idempotent, if there exisit any. $\Gamma_{1}=\operatorname{Cay}(H, S)$ and $\Gamma_{2}=\operatorname{Cay}(K, T)$ and $\Gamma=(V, E)$ be the strong product of them and we denote it by $\Gamma=\Gamma_{1} \otimes \Gamma_{2}$.

By the definition 1.1, theorem 2.1 and theorem 3.1, we conclude the following theorems.

Theorem 4.1. $\Gamma$ the strong product of two Cayley graph of semigroups is also a Cayley graph of the monoid $H^{1} \times K^{1}$ with respect to the subset $\left(\left\{1_{H^{1}}\right\} \times T\right) \cup\left(S \times\left\{1_{K^{1}}\right\}\right) \cup(S \times T)$ of $H^{1} \times K^{1}$.

Theorem 4.2. Let $\Gamma_{1}$ and $\Gamma_{2}$ be two Col-Aut-vertex transitive Cayley graphs of finite semigroups. Then $\Gamma=(V, E)$ the strong product of them is also Col-Aut-vertex transitive Cayley graph.

\section{Co-normal product}

Through this section we assume $H$ and $K$ are two semigroups, $S$ and $T$ be the subsets of $H$ and $K$ respectively which does not contain the identity and idempotent, if there exisit any. $\Gamma_{1}=\operatorname{Cay}(H, S)$ and $\Gamma_{2}=\operatorname{Cay}(K, T)$ and $\Gamma=(V, E)$ be the co-normal product of them and we denote it by $\Gamma=\Gamma_{1} \odot \Gamma_{2}$.

Theorem 5.1. $\Gamma$ the co-normal product of two Cayley graph of groups is also a Cayley graph of the group $H \times K$ with respect to the subset $(H \times T) \cup(S \times K\})$ of $H \times K$.

Proof. By definition 1.1, $\left(\left(h_{1}, k_{1}\right),\left(h_{2}, k_{2}\right)\right)$ is an edge of $\Gamma$ if and only if for some $s \in S$ we have $h_{2}=s h_{1}$ or $k_{2}=t k_{1}$ for some $t \in T$. Equivalently for some $\alpha=(s, t) \in(S \times K) \cup(H \times T)$ we have $\left(h_{2}, k_{2}\right)=\alpha\left(h_{1}, k_{1}\right)$

In the theorem 5.1, we brought a strong condition that $H$ and $K$ to be groups, because if they did not satisfy the conditions of groups then we can not say the co-normal product of the corresponding Cayley graphs will be a Cayley graph of the product semigroup $H \times K$. with respect to any subset of it. In the following example we bring a evidence of the case.

Example 5.2. Let $H$ be a semigroup and $S$ be a subset of it, $K$ be a monogenic of the semigroup generated by $x$ which satisfies the condition $x^{n+r}=x^{r}$ for some $n>2$ and $r>1$. and $T=\{x\}$.

For aome $v_{1} \in H$ and $s \in S$ set $u_{=} s v_{1}, u_{2}=x^{r}$ and $v_{2}=x$. By the definition of 1.1, $\left(u_{1}, v_{2}\right)$ is adjacent to $\left(v_{1}, v_{2}\right)$ in the graph $\Gamma$. If $\Gamma$ is a Cayley graph of the group $H \times K$ with respect to some subgroup $W$ of it, then we can say there exists $w=(h, k) \in H \times K$ such that the condition $\left(v_{1}, x\right)=(h, k)\left(u_{1}, x^{r}\right)$ holds, implying for some $k \in K$, we have $x=k x^{r}$ which is sa contradiction by the definition of the monogenic $K$.

\section{Lexicographic product}

Through this section we assume $H$ and $K$ are two semigroups, $S$ and $T$ be the subsets of $H$ and $K$ respectively which does not contain the identity and idempotent, if there exisit any. $\Gamma_{1}=\operatorname{Cay}(H, S)$ and $\Gamma_{2}=\operatorname{Cay}(K, T)$ and $\Gamma=(V, E)$ be the lexicographic product of them and we denote it by $\Gamma=\Gamma_{1}\left[\Gamma_{2}\right]$.

Theorem 6.1. $\Gamma$ the lexicographic product of two Cayley graph of groups is also a Cayley graph of the group $H \times K$ with respect to the subset $\left.\left(\left\{1_{H}\right\} \times T\right) \cup(S \times K\}\right)$ of $H \times K$.

Proof. By definition 1.1, $\left(\left(h_{1}, k_{1}\right),\left(h_{2}, k_{2}\right)\right)$ is an edge of $\Gamma$ if and only if for some $s \in S$ we have $h_{2}=s h_{1}$ or $h_{1}=h_{2}$ and $k_{2}=t k_{1}$ for some $t \in T$. Equivalently for some $\alpha=(s, t) \in(S \times K) \cup\left(\left\{1_{H}\right\} \times T\right)$ we have $\left(h_{2}, k_{2}\right)=\alpha\left(h_{1}, k_{1}\right)$

The example 5.2 also provide a counter example for the case if we replace semigroup instead of group in the theorem 6.1. 


\section{Complement}

Through this section we assume $H$ is a group and $S$ be the subset of $H$ which does not contain the identity. $\Gamma=C a y(H, S)$ and $\Gamma^{c}=(V, E)$ be the complement of it.

Theorem 7.1. $\Gamma^{c}$ the complement of the Cayley graph of a group is also a Cayley graph of the group $H$ with respect to the subset $S^{c}-\{1\}$ of $H \times K$.

Proof. By definition 1.1, $\left(h_{1}, h_{2}\right)$ is an edge of $\Gamma^{c}$ if and only if it is not an edge of $\Gamma$ and $h_{1} \neq h_{2}$, if and only if $h_{2} \neq s h_{1}$ for all $s \in S$ and $h_{2} \neq 1 h_{1}$, thus we can consider $\Gamma^{c}$ a Cayley graph of the same vertex set with respect to $S^{c}-\{1\}$.

\section{References}

[1] A. Assari and N. Hoseinzadeh. Tensor product of colour vertex transitive Cayley graphs of finite semigroups, Gen. Math. Notes, Vol. 19, No. 1, November, 2013, pp. 28-34.

[2] A. Gardiner, C. E. Praeger, On 4-valent symmetric graphs, European J. Combin., 1994, 15: 375-381.

[3] A. Gardiner, C. E. Praeger, A characterization of certain families of 4-valent sym- metric graphs, European J. Combin., 1994, 15: 383397.

[4] M. V. Lawson, Inverse semigroups: the theory of partial symmetries, World Scientific Pub- lishing Co., Inc., NJ, 1998.

[5] A. V. Kelarev and C. E. Praeger, On transitive Cayley graphs of groups and semigroups, European J. of Com. No.24 (2003) 59-72.

[6] S. Spacapan, Connectivity of Cartesian product of graphs, Applied Mathematica Letters Vol. 21, Issue 7, $2008,682-685$.

[7] F. Li , W. Wang, Z. Xu, H. Zhao. Some results on the lexicographic product of vertex-transitive graphs, Applied Math. Letters, 2011,24, 1924-1926. 